Sheridan College

SOURCE: Sheridan Institutional Repository

Publications and Scholarship

Faculty of Applied Science \& Technology (FAST)

7-29-2016

\title{
Wireless resource virtualization: opportunities, challenges, and solutions
}

M. Kali

Western University

M. Youssef

Western University

A. Shami

Western University

A. Al-Dweik

Western University

Shirook Ali

Sheridan College, shirook.ali@sheridancollege.ca

Follow this and additional works at: https://source.sheridancollege.ca/fast_publications

Part of the Electrical and Electronics Commons, and the Systems and Communications Commons

\section{SOURCE Citation}

Kali, M.; Youssef, M.; Shami, A.; Al-Dweik, A.; and Ali, Shirook, "Wireless resource virtualization: opportunities, challenges, and solutions" (2016). Publications and Scholarship. 45.

https://source.sheridancollege.ca/fast_publications/45

\section{(c) (i) $(9$}

This work is licensed under a Creative Commons Attribution-Noncommercial-No Derivative Works 4.0 License. This Article is brought to you for free and open access by the Faculty of Applied Science \& Technology (FAST) at SOURCE: Sheridan Institutional Repository. It has been accepted for inclusion in Publications and Scholarship by an authorized administrator of SOURCE: Sheridan Institutional Repository. For more information, please contact source@sheridancollege.ca. 


\title{
RESEARCH ARTICLE
}

\section{Wireless resource virtualization: opportunities, challenges, and solutions}

\author{
M. Kalil ${ }^{1 *}$, M. Youssef ${ }^{1}$, A. Shami ${ }^{1}$, A. Al-Dweik ${ }^{1,2}$ and S. Ali ${ }^{3}$ \\ 1 Western University, London, Ontario, Canada \\ 2 Department of Electrical and Computer Engineering, Khalifa University, UAE \\ ${ }^{3}$ Sheridan College, Brampton, Ontario
}

\begin{abstract}
Wireless resource virtualization (WRV) is currently emerging as a key technology to overcome the major challenges facing the mobile network operators (MNOs) such as reducing the capital, minimizing the operating expenses, improving the quality of service, and satisfying the growing demand for mobile services. Achieving such conflicting objectives simultaneously requires a highly efficient utilization of the available resources including the network infrastructure and the reserved spectrum. In this paper, the most dominant WRV frameworks are discussed where different levels of network infrastructure and spectrum resources are shared between multiple MNOs. Moreover, we summarize the major benefits and most pressing business challenges of deploying WRV. We further highlight the technical challenges and requirements for abstraction and sharing of spectrum resources in next generation networks. In addition, we provide guidelines for implementing comprehensive solutions that are able to abstract and share the spectrum resources in next generation network. The paper also presents an efficient algorithm for base station virtualization in long-term evolution (LTE) networks to share the wireless resources between MNOs who apply different scheduling polices. The proposed algorithm maintains a high-level of isolation and offers throughput performance gain. Copyright (C) 2016 John Wiley \& Sons, Ltd.
\end{abstract}

KEYWORDS

virtualization; wireless networks; resource sharing; mobile networks

*Correspondence

M. Kalil, Western University, London, Ontario, Canada.

E-mail:mkalil3@uwo.ca

\section{INTRODUCTION}

In the last decade, the demand for mobile data services has experienced a substantial growth that provoked the mobile network operators (MNOs) to search for novel solutions to satisfy the surging demand while increasing the average revenue per user. Mobile data traffic forecasts provided by companies such as Cisco estimated 11-fold increase in the global mobile data traffic between 2013 and 2018 [1]. Thus, MNOs need to stretch the capacity of their mobile networks into a new horizon to satisfy the expected demand. As the revenues generated do not commensurate with the traffic growth, operators should increase their capacity while being extremely cautious with their investment. They must efficiently utilize the scarce and highly expensive wireless resources. For example, in 2011, the Federal Communications Commission held Auction 92 in order to sell spectrum licenses in the $700 \mathrm{MHz}$ band. The seven winners paid $\$ 19.8 \mathrm{bn}$ for 16 licenses. On the other hand, recent spectrum utilization measurements have shown that wireless resources are underutilized in many cases, which urge the call for action to create innovative solutions for the problem of underutilized wireless resources.

Wireless resource virtualization (WRV) is gaining a remarkable interest by the industry as a promising solution to address the problems of spectrum scarcity and resources utilization inefficiency. WRV enables network operators to create multiple logical networks on a single physical substrate, thus yielding better efficiency in terms of energy consumption and hardware utilization as shown in Figure 1, where a single base station (BS) is shared among three MNOs. Sharing BS hardware components as well as spectrum resources are of great interest to MNOs. A recent study from ABI Research [2] shows that a worldwide active infrastructure sharing over a period of 5 years can save up to $\$ 60 \mathrm{bn}$ in capital expenditure (CAPEX) and operating expenditure (OPEX).

While virtualization of wireless networks has recently received an increased attention, the concept of virtualiza- 


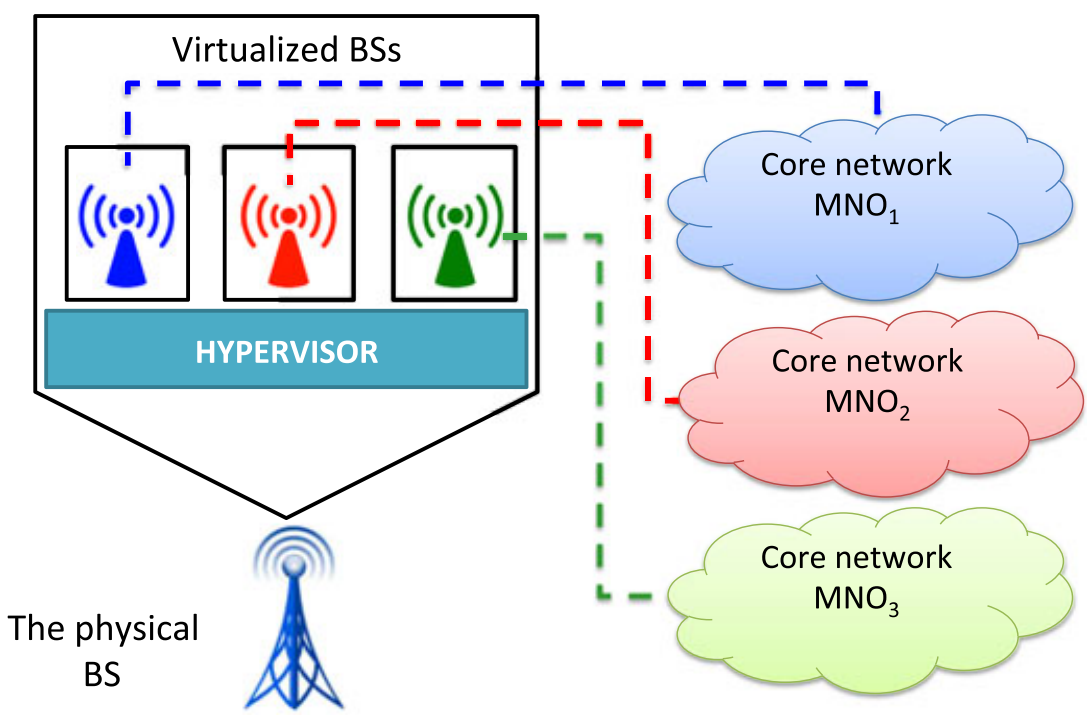

Figure 1. Base station (BS) virtualization.

tion is not new. For example, memory virtualization was introduced in 1956. The main idea is to make the application see a contiguous memory space while the actual situation behind-the-scene is that the memory is fragmented and spread across multiple primary and secondary physical memory storages. The concept has then been extended into datacenters where servers share a pool of memory to overcome limitations of physical local memory. Since then, virtualization has evolved remarkably over the past 2 decades for wired networks. Using network virtualization, software such as VMwARE NSX can be used to spin a network as easy as spinning virtual machines. Virtual networks can be saved, deleted, or restored. All network services are turned into software that uses the physical resources as an Internet Protocol (IP) backplane. The network is managed by hypervisors spread across the datacenters.

Although general concepts of network virtualization can be applied regardless of the network type, solutions from wired networks cannot be directly applied to wireless networks. The main reason is that all links in wired networks are reliable, physically isolated from each other, and have a constant bandwidth. Hence, the physical layer can often be ignored without major impact on the network performance. The same approach cannot be applied to wireless networks because of the dynamic nature of wireless channels. Wireless links are less reliable, suffer from interference, and have a fluctuating capacity depending on the channel quality. Therefore, the physical layer of wireless networks profoundly affects the network performance and should be taken into consideration during the virtualization process. Furthermore, the resources allocation process in wireless networks faces additional challenges as a result of the dynamic nature of the wireless channel, wireless network topologies, mobility of users, and fast variation of Quality of Service (QoS) requirements of on-the-go applications.
These challenges mandate the presence of a hypervisor that is capable of monitoring the resources allocation to different networks, coping with the rapid variations of the wireless channel and users' demands, and assuring the adherence to the sharing agreement between the MNOs.

The major contributions of the paper are summarized as follows:

- This work classifies the main benefits of WRV. In addition, it provides an overview of existing WRV techniques and classifies them based on their depth of sharing.

- The paper discusses the challenges in virtualizing next generation networks. Moreover, it provides guidelines for implementing comprehensive solutions that are able to share the wireless resources in a multiple radio access technology (multi-RAT) heterogeneous network (HetNet).

- A solution for BS virtualization in long-term evolution (LTE) networks to share the wireless resources between MNOs is proposed. The solution maintains a high-level of isolation between MNOs and offers throughput performance gain. It also acknowledges the varying nature of the wireless channel and makes sure that the resource blocks (RBs) are sent to the best receptors.

The remaining part of the paper is organized as follows: Sections 2 and 3 discuss the main benefits and challenges of adopting WRV. In Section 4, possible solutions for WRV are investigated. Section 5 discusses solutions for virtualizing next generation radio access networks. In Section 6, we propose and evaluate an efficient solution for BS virtualization in LTE systems, and Section 7 concludes the paper. 


\section{MAIN BENEFITS OF WIRELESS RESOURCE VIRTUALIZATION}

Wireless resource virtualization offers pivotal benefits to the wireless market as well as the environment. The main benefits of WRV can be divided into three groups as follows.

\subsection{Economic sharing of investment and cost reduction}

The most immediate benefit of WRV is the considerable reduction of the CAPEX and OPEX. By consolidating their equipments and spectrum licenses, MNOs are able to enhance their network coverage and to alleviate the capital investment needed for launching new services, which minimizes the time-to-market of new innovations. For example, an MNO does not have to solely own a certain band through expensive and uncertain bedding systems. It needs simply to get into a sharing agreement with an existing MNO who owns the required band.

When multiple network operators jointly construct a network, hardware equipment such as antennas, cooling systems, and towers can be shared, which reduces the operational costs and power consumption. Moreover, resources sharing overcomes the capital shortage related to new infrastructure investments, resulting in shorter network deployment periods and expedited time-to-market process. Additionally, WRV enables the incorporation of spectrum pooling techniques to improve the spectrum utilization and increase the total network capacity [3]. Therefore, a great economic potential is created for reaping the benefits of the growing wireless market, and it enables business innovation in mobile services and applications.

\subsection{Collaborative business models}

Governments assign spectrum licenses through competitive mechanisms such as spectrum auctions or competitive tender, which is commonly referred to as beauty contests. Because the spectrum available for transmission is limited, a small number of MNOs are usually dominating the wireless market, which increases the cost of services provided to the end users. Wireless virtualization enables the creation of a virtual MNO that purchases the spectrum usage rights from an MNO and provides wireless services to the end customers under its own brand name. Virtual MNO is a new business model that enables fair competition that benefits the end customers. Furthermore, it improves resource utilization by selling underutilized network capacity.

\subsection{Environmental benefits}

Energy consumption in wireless networks is a growing concern for MNOs because of the increasing energy prices and the environmental consequences. The information and communication technology industry currently consumes about $3 \%$ of the global energy and emits $2 \%$ of the global
Carbon dioxide $\left(\mathrm{CO}_{2}\right)$ [4]. Such numbers may rise sharply if each new MNO has to install new infrastructure for each new network.

While many works focus on virtualizing the core network [5], a significant portion of the energy consumed by cellular networks is used by the radio access network (RAN) subsystem. Thus, WRV can increase the energy efficiency of cellular networks because sharing equipment and sites between multiple MNOs reduces the number of new access nodes required by new market players or by existing operators who want to extend the coverage of their networks. Therefore, the energy consumed and the expansion of the wireless communications sector will not be growing at the same rates. Or the current MNOs can utilize the WRV to reduce the current energy consumption and the $\mathrm{CO} 2$ emissions, which promotes the green radio communication. Recently, energy consumption has become a key element in designing wireless systems and building green networks. Although most previous work has focused on power consumption at the user equipment (UE) end to extend the mobile battery life, more recent efforts have been concentrated on the wireless network energy consumption because of its economic and environmental benefits [6].

\section{OPERATIONAL AND BUSINESS CHALLENGES OF WIRELESS RESOURCE VIRTUALIZATION DEPLOYMENT}

In spite of the potential benefits and cost savings of WRV, two main operational and business challenges prevent widespread deployment of WRV:

\subsection{The risk of market share loss and anti-competitive practices}

While WRV facilitates network deployment, accelerates the penetration of start-up operators, and boosts the market competition, it exposes the well-established large operators, to the risk of market share loss. The large operators hope to achieve cost savings through infrastructure-sharing with competitors. However, it raises the fear that relaxing the requirements for new players to enter the mobile market may dilute their market shares. Consequently, large network operators will attempt to outline the WRV process to empower themselves to control the market and reduce the success chances of smaller operators.

\subsection{Independence of services with radio access network-sharing}

The operators' ability to control the network entities is essential for innovation and provision of new services. While WRV consolidates hardware and resources of multiple MNOs, it limits the operators' independence and slows down services deployment because other operators might be influenced by particular decisions. Therefore, deducing 


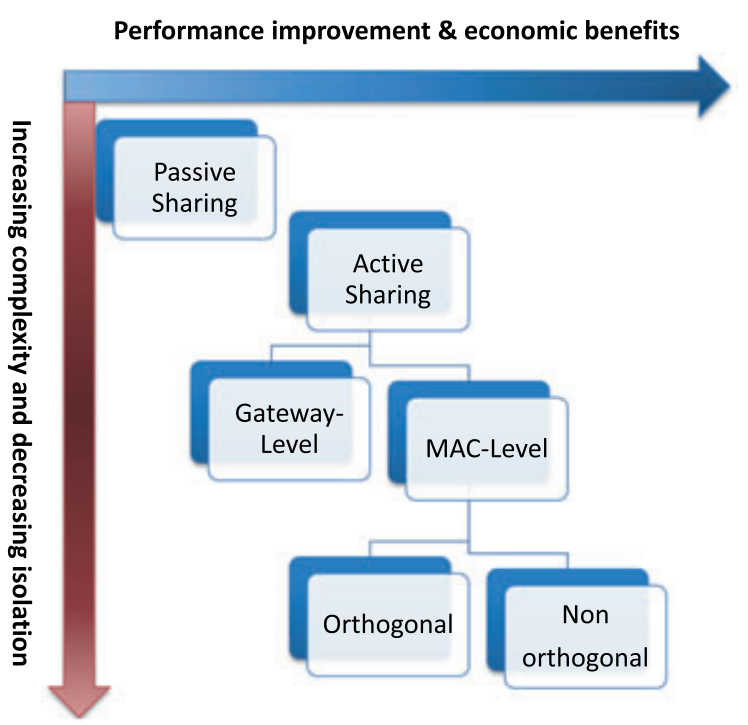

Figure 2. Scope of virtualization.

new strategies to enable the deployment of particular services for some operators in the context of WRV is still required and remains a challenging aspect for the WRV process.

\section{SCOPE OF VIRTUALIZATION AND THE DEPTH OF SHARING}

Wireless resource sharing can be achieved at different levels. Two main scopes can characterize the depth of sharing, namely, passive sharing and active sharing, as seen in Figure 2.

\subsection{Passive sharing}

In passive sharing, operators share passive infrastructure entities such as radio masts and towers, power supply, air conditioning sites, and building premises. Passive sharing has become popular since 2000 because it reduces initial investment and saves operating costs. Because no active operational coordination between operators is required, passive sharing is straightforward to apply, which makes it the most widespread sharing scope, particularly in lowdensity areas. For example, $40 \%$ of Orange sites in the rural areas of France are subject to passive sharing. Another example of passive sharing is the agreement between Vodafone and Telefonica in 2009 where they announced a wide-ranging agreement to share mobile networks' infrastructure in the UK, Spain, Ireland, and Germany to cope with the demand for mobile broadband data services and to save millions of pounds in costs [7]. However, the limitation of passive sharing is that no network capacity improvement is expected.

\subsection{Active sharing}

In active sharing, network active elements are included such as electronic infrastructure, transceivers, antennas, spectrum, and fiber optic networks. Furthermore, the sharing can be extended to reach core network components such as Mobility Management Entity. Active infrastructure sharing provides more savings in CAPEX and OPEX as compared with passive sharing. Therefore, it is receiving a proliferating attention by wireless operators and standards development organizations. The Third Generation Partnership Project LTE standard supports two active sharing configurations as depicted in Figure 3, MultiOperator Core Network and Gateway Core Network. In Multi-Operator Core Network configuration, each MNO has its own core network. However, Gateway Core Network extends the sharing to the core network, where MNOs can also share the Mobility Management Entity of the core network, which enables additional cost savings but reduces the level of isolation and dependency between MNOs. Each core network operator is identified by a distinguished Public Land Mobile Network Identification (PLMN-ID). Shared cells broadcast the PLMN-IDs of the MNOs that share the network. Each UE accesses the shared network by selecting one of available PLMN-IDs. Active sharing can be achieved at different levels. Deeper level of sharing results in higher CAPEX and OPEX reduction, but increases the deployment complexity and limits the operators freedom to manage and operate the network. Active sharing solutions can be classified into two groups based on the level of sharing, which are outlined in the following two subsections.

(1) Gateway-level solution The work reported in [2] proposes a gateway-level solution that enables multiple MNOs to share one single physical BS without modifying its medium access control (MAC) schedulers. The main advantage of such solution is the facilitation of immediate deployment of RAN sharing because no major modifications are required to existing BSs. However, the resource allocation decisions are invisible to the gateway, which degrades the performance of the sharing process. In such solutions, the virtualization happens by reshaping the traffic at the gateway level. For the downlink, the gateway controls the number of packets sent to the BS for each operator. While sending the traffic to the BS, the gateway considers sharing requirements such as efficient resource utilization and isolation between different operators. For the uplink, controlling the resource allocation is not straightforward because the traffic is generated by the end users, which cannot be directly controlled by the gateway. This can be solved by creating a feedback channel between the BS and the gateway. The gateway sets an upper-bound for the average data rate for each data flow. The gateway con- 


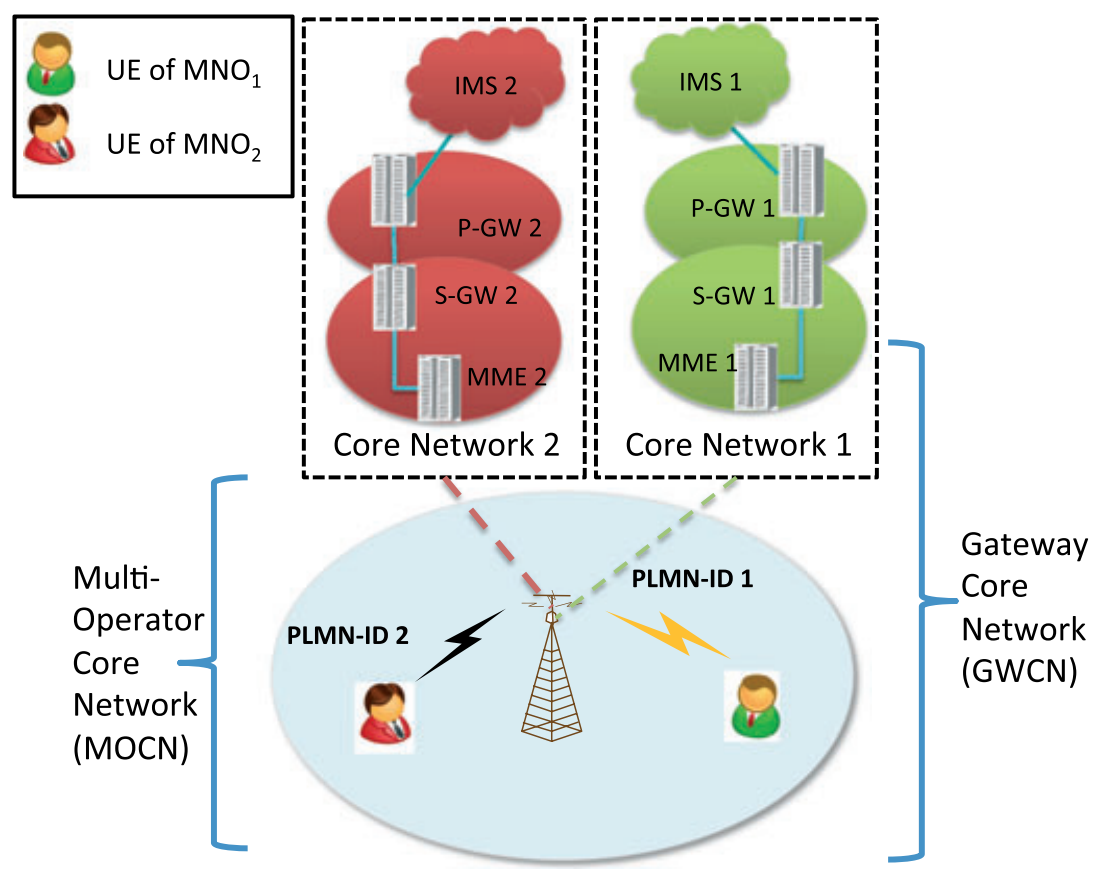

Figure 3. Long-term evolution sharing configuration options. UE, user equipment; PLMN-ID, Public Land Mobile Network Identification.

trols the uplink sharing by tuning the flows' upper bound values.

(2) MAC-level solution Virtualizing an LTE BS at the MAC level is tackled in [3] where full control of the internal scheduler of the BS is accessible. The main motivation is to provide the most efficient sharing of the wireless resources between users who belong to different MNOs. The scheduler considers various perspectives while assigning the recourses to users such as the quality variation of the wireless channel, satisfying the MNOs' service-level agreements (SLAs), efficient utilization of the wireless resources, providing tight isolation between MNOs who are sharing the same physical substrate, and enabling MNOs to implement their own custom scheduling policies that fit their service requirements and business models. This solution gives the maximum efficiency of resource utilization as the resource allocation decisions are fully controlled at the BS level. Another LTE air interface virtualization scheme is proposed in [8], where a hypervisor is added on top of the physical resources. This hypervisor is responsible of virtualizing the evolved Node-B (eNB) into a number of virtual eNBs that are then used by different MNOs. It was shown that the capacity gains can be achieved by sharing the spectrum resources between different MNOs. More practical scenarios are studied in [9] where MNOs share multiple eNBs, with the sharing process managed by the hypervisor. Enhancements such as load balancing and safety margins were investigated. A flow-level virtualization scheme of wireless resources on BSs in Worldwide Interoperability for Microwave Access network was proposed and evaluated in [10]. The BS radio resources are sliced between different flow groups, and the solution of [10] enables customized flow scheduling per slice, where each slice can be treated as a virtual MNO that supports a set of flows.

(3) Orthogonal versus non-orthogonal sharing The authors of [11] distinguish between two main sharing models:

Orthogonal sharing: multiple operators share their spectrum without introducing mutual interference. Therefore, a radio block is assigned only to one operator in any given time slot. Sharing can be (i) full: where the MNOs aggregate and share their entire spectra or (b) partial: where MNOs prefer to have sole ownership on a certain band in order to satisfy QoS guarantees for their customers. The authors of [11] show that the overall theoretical throughput gain of orthogonal sharing is about $12 \%$. Although it is not a substantial gain, it comes almost at no cost. It is just a matter of taking advantage of the asymmetry of the loads between the MNOs. Orthogonal sharing might require BS with extra capabilities to be able to accommodate a larger spectrum and a high-data backbone connection in order to convey the increased traffic.

Non-orthogonal sharing: in this scenario, a frequency band can be allocated simultaneously to two operators. This model offers much higher spectrum efficiency gains, yet it increases the complexity of the system because 
interference reduction or cancellation techniques should be incorporated. The enablers to manage the interference between different BSs to improve the overall system performance are discussed in [11]. The main enabler is called transmit beamforming where a BS with multiple antennas can steer the transmission power towards a certain destination using the appropriate scaling of the transmitted signal in each antenna. Hence, interference is managed by spatial separation rather than time or frequency separation as in time division multiple access (TDMA) or frequency division multiple access (FDMA). In order to take advantage of beamforming, the MNOs have to exchange channel state information via an appropriate feedback interface.

\section{VIRTUALIZATION IN NEXT GENERATION RADIO ACCESS NETWORK}

A key enabler of next generation network such as 5G is the integration of multiple RATs including 4G, 3G (Universal Mobile Telecommunications System (UMTS), and High-Speed Packet Access (HSPA)), General Packet Radio Service (GPRS) and Enhanced Data rates for Global Evolution (EDGE) and Wi-Fi. Multi-RAT network performance can be improved by smartly utilizing the wireless resources available among each RAT [12].

HetNets have emerged as a new network planning drift. It is expected to play a vital role to improve the overall customer experience. It is currently considered as a promising solution to satisfy the growing demand for broadband wireless access networks. HetNets consist of a mix of technologies, frequencies, cell sizes, and network architectures to enhance capacity and coverage of wireless networks. Virtualizing the RAN into a number of virtual RANs is determined by the procedure by which the resources are scheduled, controlled, and shared between users who belong to different operators across such multiRAT HetNets. The resource scheduler should be able to individually virtualize both uplink and downlink resources while maintain QoS and sharing requirements. For example, as macrocells transmit at high power-levels, UEs can be connected to a macrocell in the downlink. However, the transmit power of UEs is limited to a small-size battery. Hence, connecting the UEs to a picocell in the uplink enables the UE to transmit at much lower power levels because of the short distance between the UE and the BS.

A comprehensive solution for virtualizing next generation RANs should consider both intra-BS and inter-BS scheduling as illustrated in Figure 4 where a BS can be of any size and applies any RAT.

The intra-BS scheduling aims at virtualizing a BS into a number of virtual BSs, each of which can be used by a different MNO. Solutions for resource allocation in wireless system have been extensively investigated in the literature [13]. However, these solutions consider only allocating resources to users belonging to the same operator. Sharing the resources between multiple operators adds an extra dimension to the allocation problem, which is scheduling the resources across MNOs who have different QoS requirements in addition to scheduling the resources between users.

In general, there are two main approaches to solve the intra-BS problem. The first approach allows the direct application of the schedulers that already exist in the literature by dividing the allocation problem into two subproblems. The first subproblem is concerned with dividing the resources between MNOs based on the traffic load and the sharing agreement. Then, MNOs are allowed to allocate wireless resource within their share using traditional schedulers. This solution requires a fair and accurate tool to estimate the traffic load of each operator. What differentiates this solution from the static sharing (SS) scenario is that the number of resources assigned to each MNO changes periodically based on the traffic load and the sharing agreement. As the resources are divided across MNOs regardless of the users channel conditions, and prior to scheduling the resources to the users, the multi-operator gain is not perfectly utilized. However, a high level of isolation between MNOs is maintained. An example of this solution is given in [9].

The second approach considers the intra-BS resource allocation as one problem to fully utilize the multi-operator gain. The scheduler should consider various perspectives such as wireless channel quality, satisfying the MNOs' SLA, maintaining QoS requirements, providing tight isolation between MNOs, and enabling MNOs to implement the scheduling policies that fit their service requirements and business models. This solution compromises isolation between MNOs in favor of efficient usage of resources. An example of this solution is presented in [6], where the problem is presented as a utility-based allocation problem.

The inter-BS scheduler addresses effective scheduling of wireless resources pertaining to multiple eNBs that are shared between multiple MNOs. The inter-BS solution adds another dimension to the optimization problem that should consider the entire system of users, individual BSs, and the aggregated performance of all BSs. Orthogonal and non-orthogonal configurations can be used while distributing the resources across BSs. Orthogonal sharing assigns exclusive frequency bands to each cell sector. How-

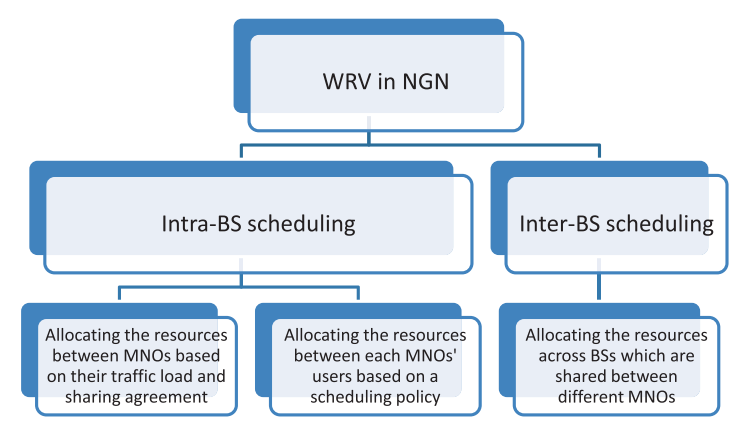

Figure 4. Scheduling tasks that should be considered for NGN virtualization. NGN, next generation network; WRV, wireless resource virtualization; BS, base station; MNOs, network operators. 
ever, non-orthogonal sharing allows neighbor sectors to share the same frequency bands. Non-orthogonal sharing necessitates the coordination between neighbored cells in order to reduce intercell interference. For example, LTE uses fractional frequency reuse approaches to control intercell interference of the cell edge users. With fractional frequency reuse, users are assigned different parts of the wireless resources based on their location in the cell. Users who are close to the center of the cell are subject to low-intercell interference from neighbored cells and are allowed to use the entire frequency band (frequency reuse factor of one). However, users at cell edges are subject to high-intercell interference. Exclusive frequency bands that are not used in adjacent cells are assigned to cell-edge users to reduce the intercell interference.

As inter-BS requires communication and coordination between different BSs, Cloud-RAN (C-RAN) is a promising enabler for inter-BS scheduling problem. C-RAN is an emerging platform that creates centralized processing pool or "cloud"of RANs [14]. The key concept of C-RAN is to separate the remote radio units from the processing function unit of BSs. BS processing functions and resources are delegated to base-band unit (BBU) pool that is implemented on "inexpensive" real-time cloud in remote datacenters. In opposition to traditional RAN where BSs are sized based on the expected peak load, C-RAN makes it feasible to select the dimensions of single cells based on average figures. During peak hours, the required processing power can be pooled and assigned on-demand basis. Typically, the BS average load is quite low compared with the peak load and varies across different areas. For example, the peak loads in residential areas occur during the night time, whereas peak loads in central office areas occur during work hours. Therefore, centralizing the processing power in BBUs results in a better utilization of the processing resources and requires fewer BBUs as compared with the traditional RAN architecture. C-RAN provides a multitude of benefits to MNOs such as the significant CAPEX and OPEX savings, green infrastructure, and easier traffic load balancing.

\section{PROPOSED SOLUTION FOR VIRTUALIZING EVOLVED NODE-B IN LONG-TERM EVOLUTION SYSTEM}

In this section, we propose a solution for virtualizing an eNB that serves users of two different operators. The RBs of both MNOs are pooled at the eNB. We assume that $\mathrm{MNO}_{1}$ has the license for a spectrum band that consists of RBs numbers $1,2,3, \ldots, M_{1}$, while $\mathrm{MNO}_{2}$ has the license of a spectrum band that contains the RBs numbers $M_{1}+1, M_{1}+2, M_{1}+3, \ldots, M$. The focus here is to provide a solution that allows operators to efficiently share the resources with a high level of independence. We divide the resource scheduler task into two steps as depicted in Figure 5. In the first step, the operators are fully independent, no sharing is applied, and each operator has full control over its resources. Each operator is free to apply any scheduling policy that fits its business model and SLA. In the literature, various scheduling policies are presented. For example, naive schedulers such as round robin (RR), QoS-aware schedulers such as priority set scheduler, channel-aware schedulers such as the proportional fair (PF) and maximum throughput (MT), channeland queue-aware schedulers such as the modified largest weighted delay first, exponential PF, EXP rule, and LOG rule [13].

In the second step of the resource scheduling process, the allocation decisions of the first step are further processed by performing two main processes as follows:

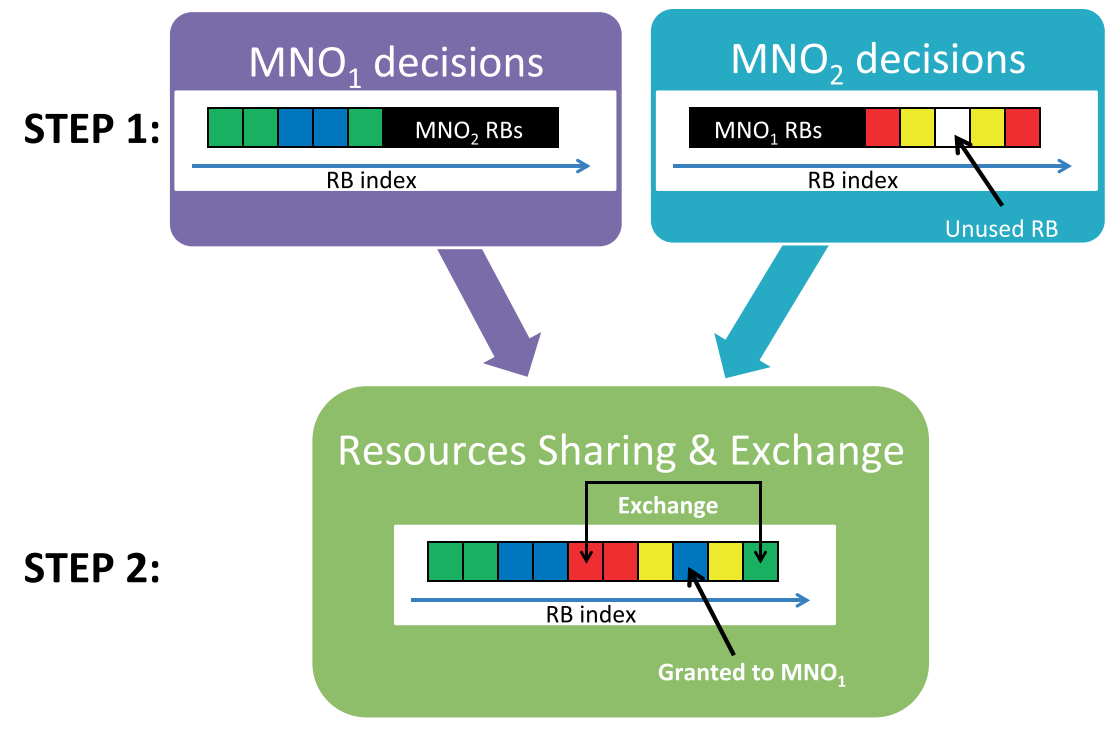

Figure 5. Evolved Node-B virtualization architecture. MNO, network operator; RB, resource block. 
First, considering that $\mathrm{MNO}_{1}$ is the operator with low traffic, the unused $\mathrm{RBs}$ of $\mathrm{MNO}_{1}$ are assigned to users of $\mathrm{MNO}_{2}$ according to the scheduling policy of $\mathrm{MNO}_{2}$. In fact, the event of having unused RBs is more likely to happen in the uplink transmission because of the limited transmit power in the mobile devices. In LTE systems, the transmission power is equally divided between the assigned RBs to a user. As more RBs are assigned to a user, the transmit power per RB decreases, which may reduce the received signal-to-noise (SNR). Therefore, assigning more RBs for a user in the uplink does not always increase the transmission rate of the user.

Second, because wireless channels are subject to multipath fading and Doppler spread, the channels are timevarying and frequency selective. Therefore, the quality of a particular RB will be seen different by different. Accordingly, users of $\mathrm{MNO}_{1}$ can exchange RBs of $\mathrm{MNO}_{2}$ if they are of better quality for them and vice versa. Consequently, the scheduler provides the maximum resource utilization while keeping the operators independent.

\subsection{Evaluation}

We evaluate the performance of the considered system using a MATLAB system-level simulation. The block resources are shared based on SS and dynamic sharing (DS) polices. The SS scenario is a passive sharing where each MNO individually allocates its own RBs to its users based on its scheduling policy. Users who belong to $\mathrm{MNO}_{1}$ cannot be assigned RBs that belong to $\mathrm{MNO}_{2}$ spectrum band and vice versa. For the DS, any RB at the eNB can be allocated to any user. It is assumed that $\mathrm{MNO}_{1}$ applies PF scheduling and serves 20 users. The first 10 users numbered $1,2, \ldots, 10$ are far from the cell center and have an average SNR of $10 \mathrm{~dB}$. Users $11, \ldots, 20$ are closer to the cell center and experience an average SNR of $20 \mathrm{~dB}$. For $\mathrm{MNO}_{2}$, the scheduling policies and number of users who subscribed to the network are different from those of $\mathrm{MNO}_{1}$ to cover more scenarios. A backlogged traffic model is considered where users always have packets to transmit. Each user is assumed to have a non guaranteed bit rate bearer with an aggregate maximum bit rate of $1 \mathrm{Mbps}$.

Figure 6 compares the performance of SS with the DS in terms of throughput. $\mathrm{MNO}_{2}$ is assumed to apply MT

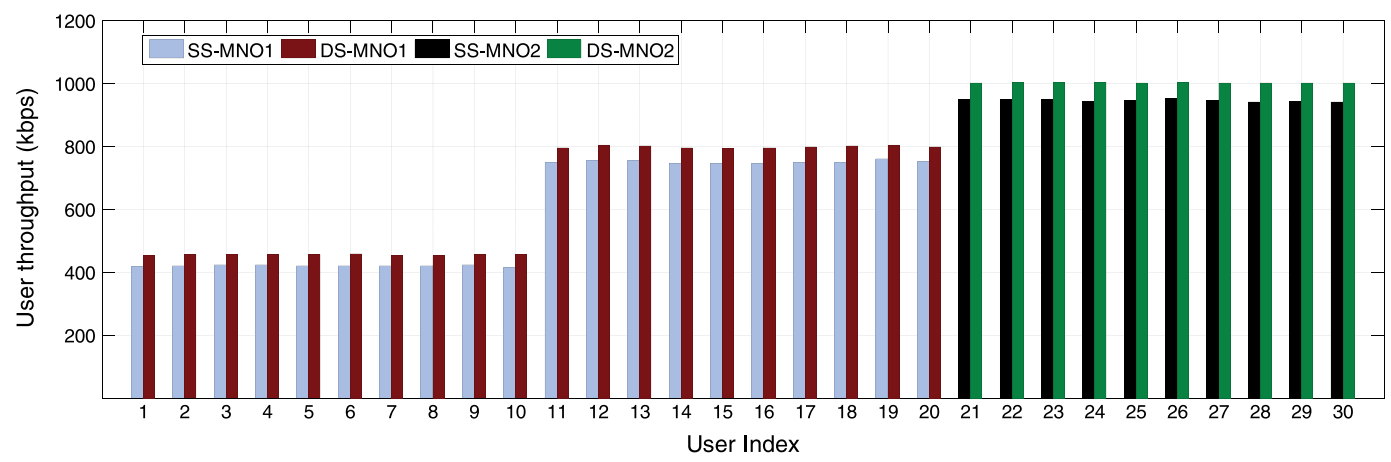

Figure 6. Performance evaluation of the proposed WRV. $\mathrm{MNO}_{1}$ applies PF scheduling while $\mathrm{MNO}_{2}$ applies MT scheduling. Users number 1:10 belong to $\mathrm{MNO}_{1}$ and receive an average SNR of $5 \mathrm{~dB}$. Users number 11:20 belong to $\mathrm{MNO}_{1}$ and receive an average SNR of $10 \mathrm{~dB}$. Users number 21:30 belong to $\mathrm{MNO}_{2}$ and receive an average SNR of $10 \mathrm{~dB}$.

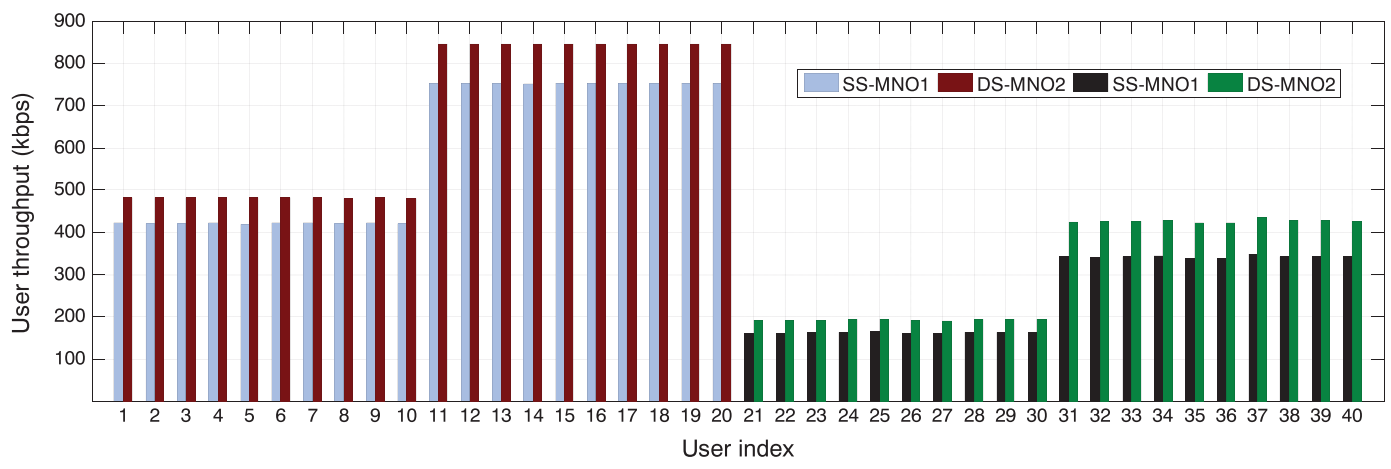

Figure 7. Performance evaluation of the proposed WRV. $\mathrm{MNO}_{1}$ applies $\mathrm{PF}$ scheduling while $\mathrm{MNO}_{1}$ applies RR scheduling. Users number 1:10 belong to $\mathrm{MNO}_{1}$ and receive an average SNR of $5 \mathrm{~dB}$. Users number 11:20 belong to $\mathrm{MNO}_{1}$ and receive an average SNR of $10 \mathrm{~dB}$. Users number 21:30 belong to $\mathrm{MNO}_{2}$ and receive an average SNR of $5 \mathrm{~dB}$. Users number 31:40 belong to $\mathrm{MNO}_{2}$ and receive an average SNR of $10 \mathrm{~dB}$. 
scheduling and serves 10 users who receive an average SNR of $10 \mathrm{~dB}$. In case of SS, all users receive less than aggregate maximum bit rate, users 1 to 10 receive on average $0.75 \mathrm{Mbps}$ whereas users 11 to 20 receive on average $0.87 \mathrm{Mbps}$ as a result of better SNR. Users 21 to 30 belong to $\mathrm{MNO}_{2}$, and they receive throughput of $0.94 \mathrm{Mbps}$. In case of DS, both $\mathrm{MNO}_{1}$ and $\mathrm{MNO}_{2}$ users' throughput improves.

To further evaluate the proposed virtualization scheme, the scheduling policy of $\mathrm{MNO}_{2}$ is changed to $\mathrm{RR}$, and the number of users is doubled. A comparison of SS and DS scenarios is presented in Figure 7. In DS scenario, the throughput of every user is improved compared with SS scenario. It is worth noting that PF scheduling policy considers the channel quality while allocating the resources. However, RR divides each RB into different time slots that are allocated equally between users regardless of their channel quality. Therefore, $\mathrm{MNO}_{1}$ 's users achieve higher throughput than $\mathrm{MNO}_{2}$ users as shown in Figure 7.

\section{CONCLUSION}

In this paper, we demonstrate the concepts and benefits of the WRV. In addition, scope and depth of WRV are tackled with different solutions. The obtained results revealed that deep resource sharing enhances the resources utilization efficiency. However, the network becomes more complex, and the isolation between the operators sharing the same network decreases. Moreover, we discuss the design requirements to virtualize the next generation RANs, which include HetNets with multiple radio access technologies. Furthermore, we propose a solution that virtualizes an eNB in an LTE system between users of multiple mobile operators. The RBs are assigned to users based on their conditions such that it maximizes the system overall gain. In addition, a full isolation is maintained between operators so that each operator obtains at least the same resources as in the SS scenario. The proposed solution can be easily integrated in a more comprehensive solution that considers the inter-BS scheduling as well as the virtualization of other parts of the mobile network. The system performance is evaluated using MATLAB, and the results show performance improvement while full isolation is maintained between operators.

\section{REFERENCES}

1. Cisco. Cisco visual networking index: global mobile data traffic forecast update, 2013, 2013-2018.

2. Kokku R, Mahindra R, Zhang H, Rangarajan S. Cellslice: Cellular wireless resource slicing for active RAN sharing. In Proc. 5th Int. Conf. Commun. Syst. Netw. (COMSNETS), Bangalore, 2013; 1-10.

3. Kalil M, Shami A, Ye Y. Wireless resources virtualization in LTE systems, in. In Proc IEEE Conf. Comput.
Commun. Workshops (INFOCOM WKSHPS), Toronto, ON, 2014; 363-368.

4. Ismail M, Zhuang W. Green radio communications in a heterogeneous wireless medium. IEEE Wireless Communications 2014; 21(3): 128-135.

5. H. Hawilo, A. Shami, M. Mirahmadi, R. Asal. NFV: state of the art, challenges, and implementation in next generation mobile networks (vEPC). In IEEE Network 2014; 28 (6): 18-26, DOI:10.1109/MNET.2014.6963800.

6. Hu R, Qian Y. An energy efficient and spectrum efficient wireless heterogeneous network framework for 5G systems. IEEE Communications Magazine 2014; 52(5): 94-101.

7. Orange. Orange fast facts - networks, 2013.

8. Zaki Y, Zhao L, Goerg C, Timm-Giel A. LTE wireless virtualization and spectrum management. In Proc. $3 \mathrm{rd}$ Joint IFIP Wireless and Mobile Netw. Conf. (WMNC), Budapest, 2010; 1-6.

9. Li M, Zhao L, Li X, Li X, Zaki Y, Timm-Giel A, Gorg C. Investigation of network virtualization and load balancing techniques in LTE networks, in Proc. In IEEE 75th Veh. Technol. Conf. (VTC Spring), Yokohama, 2012; 1-5.

10. Kokku R, Mahindra R, Zhang H, Rangarajan S. NVS: a substrate for virtualizing wireless resources in cellular networks. IEEE/ACM Transactions on Networking 2012; 20(5): 1333-1346.

11. Leonardo B, Torsten F, Francesco G, Eleftherios $\mathrm{K}$, Remcom L, Osman A, Irene P, Michal S, Haibin Z. Resource sharing among wireless network operators: spectrum, infrastructure, and full radio access network sharing. In SAPHYRE White Paper \#3. SAPHYRE consortium 201303-13, 2013. http://www.saphyre.eu/publications/ book-book-chapter-thesis-publications/copy_of_ saphyre-white-paper/copy_of_saphyre-white-paper. pdf.

12. Lim G, Xiong C, Cimini L J, Li G Y. Energy-efficient resource allocation for OFDMA-based multi-RAT networks. IEEE Transactions on Wireless Communications 2014; 13(5): 2696-2705.

13. Capozzi F, Piro G, Grieco L, Boggia G, Camarda P. Downlink packet scheduling in lte cellular networks: key design issues and a survey. IEEE Communications Surveys \& Tutorials 2013; 15(2): 678-700, Second.

14. K. A. Meerja, A. Shami, A. Refaey. Hailing cloud empowered radio access networks. In IEEE Wireless Communications 2015; 22 (1): 122-129, DOI:10.1109/MWC.2015.7054727. 


\section{AUTHORS' BIOGRAPHIES}

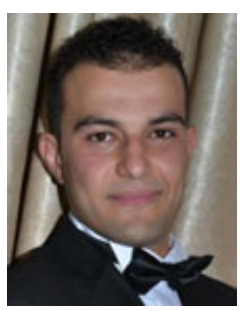

Mohamad Kalil received his BSc and MSc degrees in Electrical Engineering from Jordan University of Science and Technology, Jordan, in 2009 and 2011, respectively, and the $\mathrm{PhD}$ degree in Electrical Engineering from Western University, London, Ontario, Canada, in February 2016. Dr. Kalil is currently working as software engineer for Bluecat Networks, Toronto, Ontario, Canada. His research interests include cross-layer design, radio resource management, wireless network virtualization, and DNS security.

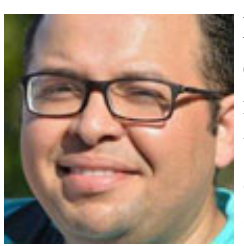

Mohamed Youssef received his BEng degree in Electrical and Telecommunications Engineering from the University of Alexandria, Egypt, in 2003, and his Master's degree of Applied Science in Electrical Engineering and Computer Sciences from Université de Sherbrooke, Canada, in 2006. Mohamed's academic interests are in data networks, wireless communications, and cloud computing. Mohamed worked in various roles in engineering and business management. For several years, Mohamed worked in Orange Business Services managing Data Networks, Data Centers, and Hosting services. Mohamed has also over 10 years of experience in the business process outsourcing (BPO) industry. He is currently working for Sutherland Global Services as a Business Operations Manager for the Canadian operations.

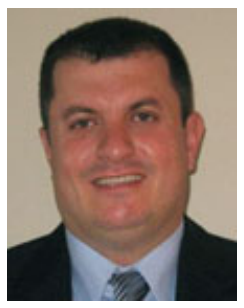

Abdallah Shami received his BE degree in Electrical and Computer Engineering from the Lebanese University, Beirut, Lebanon, in 1997, and the $\mathrm{PhD}$ degree in Electrical Engineering from the Graduate School and University Center, City University of New York, New York, NY, in September 2002. Since July 2004, he has been with Western University, Canada, where he is currently a Professor in the Department of Electrical and Computer Engineering. His current research interests are in the area of network-based cloud computing and wireless/data networking. Dr. Shami is currently an Associate Editor for IEEE Communications Survey and Tutorials, IET Communications Journal and Wiley Journal of Wireless Communications and Mobile Computing. Dr. Shami has chaired key symposia for IEEE GLOBECOM, IEEE ICC, IEEE ICNC, and ICCIT. Dr. Shami is a Senior Member of IEEE and the Chair of the IEEE Communications Society Technical Committee on Communications Software.

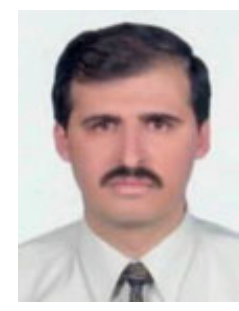

Arafat Al-Dweik received the MSc and $\mathrm{PhD}$ degrees in Electrical Engineering from Cleveland State University, Cleveland, OH, USA, in 1998 and 2001, respectively. From 2001-2003, he worked as an Assistant Professor and Chair of the Communications Technology Department at the Arab American University, Jeneen, Palestine. From 2003 until present, he was an Assistant and then Associate Professor at the Department of Electrical and Computer Engineering, Khalifa University, UAE. Moreover, he is a Research Fellow at Newcastle University, Newcastle upon Tyne, UK and Adjunct Professor at Western University, London, ON, Canada. Dr. Al-Dweik has several years of industrial experience in the USA, recipient of the Fulbright Scholarship, and has been awarded several awards and research grants. $\mathrm{He}$ is also a Senior Member of the IEEE and Associate Editor of the IEEE Transactions on Vehicular Technology and IET Communication. The main research interests of Dr. Al-Dweik include wireless communications, synchronization techniques, OFDM technology, modeling and simulation of communication systems, error control coding, and spread spectrum systems.

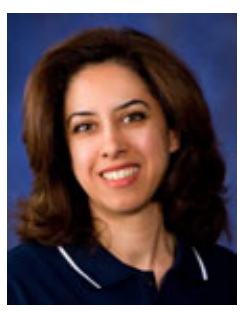

Shirook Ali has extensive and diversified engineering experience in applied research and development, teaching, innovation, knowledge transfer, and partnership from her years in academia and in industry. Prior to joining Sheridan College, Shirook led advanced research and technology teams in the high-technology industry of wireless mobile communication working at Huawei Technologies and BlackBerry Limited. Shirook advanced technologies in areas such as smart antennas and related topics for handset applications, radio frequency $(\mathrm{RF})$, channel modeling and propagation, multiple-input multiple-output (MIMO) antenna systems for $4 \mathrm{G}$ and $5 \mathrm{G}$ technologies, mmWave, tunable devices and systems, smart energy/gird, ITS, and the Internet of Things (IoT). Shirook holds 22 US and international patents in addition to several pending patents in the areas of mobile communication. She has authored more than 60 peer-reviewed journal and conference papers as well as a book chapter in the area of wireless communication. Shirook contributed a series of RAN4 contributions in 3GPP standards. Shirook is an Adjunct Professor at McMaster University, University of Western Ontario, University of Waterloo, and Guelph University. She has been awarded an NSERC Discovery Grant in support of her research in the area of nanotechnology and energy harvesting. Shirook holds BSc, MSc, and PhD degrees. She also holds the Ontario Professional Engineering designation (PEng). 